\title{
DBR3S: Um Mecanismo de Controle de Qualidade Multimídia em Redes Móveis Definidas por Software
}

\author{
Charles H. F. dos Santos ${ }^{1}$, Felipe S. Dantas Silva ${ }^{1,2}$, Augusto Venâncio Neto ${ }^{2,3}$ \\ ${ }^{1}$ Laboratório de Tecnologias Avançadas em Redes de Computadores (LaTARC) \\ Instituto Federal de Educação, Ciência e Tecnologia do Rio Grande do Norte (IFRN) \\ Natal-RN, Brasil \\ ${ }^{2}$ Departamento de Informática e Matemática Aplicada (DIMAp) \\ Universidade Federal do Rio Grande do Norte (UFRN) - Natal-RN, Brasil \\ \{charles.hallan, felipe.dantas\}@ifrn.edu.br, augusto@dimap.ufrn.br
}

\begin{abstract}
This work proposes the Dynamic Bit Rate Streaming Service over SDMN (DBR3S), a framework based on Software Defined Mobile Networks (SDMN) that implements a multimedia rate adaptation model through an optimized SDMN controller. The primary purpose of the DBR3S is to overcome the main difficulties introduced by traditional adaptive models, which are based on client implementations. The evaluations demonstrated the feasibility of DBR3S, substantially optimizing mobile users' QoE over traditional video transmission services.
\end{abstract}

Resumo. Este trabalho propõe o Dynamic Bit Rate Streaming Service over SDMN (DBR3S), um framework baseado em Redes Móveis Definidas por Software (SDMN) que implementa um modelo de adaptação de qualidade multimídia por meio de um plano de controle SDMN otimizado. O objetivo principal do DBR3S é superar as principais dificuldades introduzidas pelos modelos adaptativos tradicionais, que se baseiam em implementações nos clientes. As avaliações demonstraram a viabilidade do DBR3S, otimizando substancialmente a QoE dos usuários móveis em relação a serviços de transmissão de vídeo tradicionais.

\section{Introdução}

A popularização dos sistemas de comunicações móveis e a proliferação de dispositivos com capacidade de conexão múltipla (multihoming [Rebay 2011]), bem como o surgimento do paradigma da Internet das Coisas (Internet of Things - IoT), que visa conectar vários tipos de Objetos Móveis (Moving Objects - MOs), como carros, drones, barcos e equipamentos pessoais (e.g. smartphones e sensores), criou novas demandas de conexão móvel ininterrupta e orientada à qualidade com padrões de mobilidade e requisitos diversos (e.g. largura de banda mínima, tolerância a atraso, perda de pacotes e variação do atraso etc.). Neste cenário, arquiteturas de Redes sem Fio para Objetos Móveis (Wireless Networking for Moving Objects - WiNeMO) [Ganchev et al. 2014] são empregadas para possibilitar o atendimento dessas demandas, necessárias para fornecer o suporte adequado ao gerenciamento eficiente de recursos, um dos principais desafios na concepção de infraestruturas de redes móveis na Internet do Futuro (Future Internet - FI) [Silva et al. 2014]. 
Sob estas circunstâncias, a alta granularidade gerada pela diversidade dos requisitos das aplicações e necessidades/preferências dos usuários requer a adoção de abordagens inovadoras e sofisticadas na gerência da infraestrutura. Para atingir este objetivo, o uso de técnicas apoiadas no Gerenciamento de Rede Baseado em Políticas (Policybased Network Management - PBNM) [Strassner 2003] torna-se fundamental para prover a Qualidade de Serviço (Quality of Service - QoS) necessária para otimizar a Qualidade de Experiência (Quality of Experience - QoE) dos usuários finais [Chen et al. 2015]. Sob o ponto de vista do cliente, tais técnicas devem ser compatíveis com os Acordos de Nível de Serviço (Service Level Agreements - SLA), que descrevem, de maneira explícita, as garantias que os ISPs devem fornecer a um usuário sob determinadas circunstâncias.

De uma forma geral, é imprescindível que as capacidades apropriadas sejam agregadas à infraestrutura, de modo que o plano de controle possa ser otimizado a fim de prover perspectivas de escalabilidade e eficiência, ao mesmo tempo em que fornece novos serviços. Sob essa perspectiva, o paradigma de Redes Definidas por Software (SoftwareDefined Networking - SDN) [Haleplidis et al. 2015] vem ganhando destaque por viabilizar o desenvolvimento de novos serviços e funções de rede sem a necessidade de atualizações nos equipamentos. Com base nisso, várias soluções foram desenvolvidas para otimizar a utilização dos recursos de rede sob o paradigma SDN, como o uso de técnicas avançadas de reserva de recursos. No entanto, devido à dinamicidade que tais técnicas podem impor para comportar novas sessões, o desempenho de determinadas aplicações que possuem requisitos estáticos podem ser prejudicados. Para contornar essa limitação em aplicações multimídia, várias soluções baseadas em transmissão adaptativa (Adaptive Bit Rate Streaming - ABR) [Seufert et al. 2015] foram propostas para adaptar a taxa de transmissão das sessões às restrições das garantias de QoS, flexibilizando os requisitos das sessões e evitando que a qualidade do conteúdo transmitido seja severamente impactada pela escassez de recursos.

No entanto, as soluções tradicionais de multimídia baseadas em ABR concentram o mecanismo de controle de qualidade no aplicativo (player) cliente, o que pode resultar em dificuldades (como subutilização e instabilidade) no compartilhamento de recursos com outras sessões multimídia [Akhshabi et al. 2012]. Isso ocorre principalmente porque os players estão limitados ao escopo da interface local, fazendo com que o algoritmo de adaptação realize medições imprecisas a respeito dos recursos disponíveis. Mecanismos de adaptação orientado pelo cliente (client-driven) também podem causar um impacto significativo na performance do sistema, especialmente em equipamentos com restrição de bateria e de processamento, tais como dispositivos IoT/MOs [Gupta et al. 2012].

Nesse contexto, este trabalho propõe o Dynamic Bit Rate Streaming Service over SDMN (DBR3S), um sistema holístico de gerenciamento dinâmico de recursos para infraestruturas baseadas em SDMN, capaz de realizar o controle adaptativo de qualidade de sessões multimídia móveis de forma dinâmica. O sistema DBR3S é dotado de um conjunto de componentes funcionais que interagem para suportar serviços sofisticados, permitindo que eles gerenciem a infraestrutura de forma transparente e auto-organizável.

Por meio da abordagem centralizada do paradigma de Redes Móveis Definidas por Software (Software-Defined Mobile Networking - SDMN) [Liyanage et al. 2015], DBR3S adota um mecanismo avançado de alocação de recursos e uma interface de controle entre o controlador DBR3S (representado pelo ISP) e o servidor multimídia 
(representado pelo CDN) de modo a gerenciar dinamicamente a qualidade das sessões móveis. Isso permite que o DBR3S dissocie o mecanismo de controle de qualidade dos players clientes, distribuindo-o entre o controlador e o servidor multimídia, otimizando o compartilhamento de recursos e reduzindo a sobrecarga de processamento nos dispositivos móveis.

A partir disso, DBR3S é capaz de orquestrar um conjunto de funcionalidades, de modo a prover: $(i)$ estabelecimento de regras de QoS de acordo com as políticas de PBNM; (ii) monitoramento e aplicação de modificações nas reservas de recursos para preservar o cumprimento das definições de SLA; (iii) assistência na mobilidade dos usuários; (iv) ajuste das sessões multimídia de acordo com as regras de SLA; e (v) prover um serviço otimizado com baixa sobrecarga de processamento nos MOs.

O restante deste trabalho está organizado da seguinte maneira: a Seção 2 aponta os trabalhos relacionados ao tema de pesquisa descrito, de modo a delinear as contribuições desta proposta; a Seção 3 apresenta o a arquitetura do sistema DBR3S, expondo seus principais componentes e funcionalidades; a Seção 4 descreve em detalhes a metodologia utilizada na avaliação do sistema e apresenta uma discussão dos resultados das avaliações; a Seção 5 apresenta as considerações finais e aponta direções para trabalhos futuros.

\section{Trabalhos Relacionados}

Trabalho anterior do nosso grupo de pesquisa [Santos et al. 2017] realizou um estudo sobre multimídia adaptativa com controle de reservas QoS baseado em PBMN. A Tabela 2 apresenta uma síntese das propostas avaliadas em comparação a uma série de itens considerados como de extrema importância para compor um sistema verdadeiramente robusto de adaptação de conteúdo multimídia. Para efeito de legenda, a relação abaixo apresenta uma descrição de cada item utilizado como parâmetro de comparação:

1. QoS: Prover mecanismo de alocação de recursos ou agregação de tráfego para otimizar a transmissão de dados pela rede.

2. QoS baseado em PBNM: Prover mecanismo de QoS baseado em políticas (e.g. políticas de níveis de serviço).

3. Gerenciamento de mobilidade orientado à qualidade: Prover mecanismo de suporte a handover transparente baseado nos requisitos das aplicações.

4. Adaptação multimídia em tempo real: Prover mecanismo de controle de taxa de transmissão multimídia, adaptando a sessão às condições da rede.

5. Cooperação CDN-ISP: Prover comunicação entre os elementos da infraestrutura do ISP e os servidores do CDN, de modo a obter otimização dos níveis de eficiência na utilização da rede.

Conforme detalhado na Tabela 1, que reúne o estado da arte atualmente disponível na literatura, e sumariza os estudos realizados em trabalhos relacionados, foi constatado de que não há evidências de trabalhos anteriores que tenham se comprometido em conceber um sistema de gerenciamento de recursos capaz de realizar o controle adaptativo de qualidade de sessões multimídia móveis de forma dinâmica e transparente, reunindo uma série de recursos que permitem essa funcionalidade.

\section{Arquitetura do DBR3S}

O Dynamic Bit Rate Streaming Service over SDMN (DBR3S) tem como objetivo prover um serviço multimídia com baixos requisitos de processamento aos dispositivos clientes 


\begin{tabular}{|c|c|c|c|c|c|}
\hline Proposta & $\mathbf{\# 1}$ & $\mathbf{\# 2}$ & $\mathbf{\# 3}$ & $\mathbf{\# 4}$ & $\mathbf{\# 5}$ \\
\hline $\begin{array}{c}\text { (WALLNER; CANNISTRA, 2013), (YAN et al., 2015) } \\
\text { (TOMOVIC et al., 2014), (LOGOTA et al.,2013), (NAM et al., 2014) }\end{array}$ & $\mathrm{X}$ & & & & \\
\hline (SILVA et al., 2016) & & & & & \\
\hline (BARI et al., 2013), (MACHADO et al., 2014), (MACHADO et al., 2017) & $\mathrm{X}$ & $\mathrm{X}$ & & & \\
\hline (BENTALEB et al., 2016), (ELKHATIB et al., 2013) & $\mathrm{X}$ & & & $\mathrm{X}$ & \\
\hline (FRANK et al., 2013), (REINECKE et al., 2015) & $\mathrm{X}$ & & & & $\mathrm{X}$ \\
\hline DBR3S & $\mathrm{X}$ & $\mathrm{X}$ & $\mathrm{X}$ & $\mathrm{X}$ & $\mathrm{X}$ \\
\hline
\end{tabular}

Tabela 1. Comparativo entre os trabalhos relacionados

e, ao mesmo tempo, fornecer a melhor experiência que combine com o SLA definido entre os usuários e os ISPs. Para esse fim, o DBR3S separa o mecanismo de controle de qualidade do player cliente e o distribui entre o controlador DBR3S e o Servidor Multimídia DBR3S (DBR3S Multimedia Server - DMS). A Figura 1 expõe a arquitetura de referência do DBR3S e destaca seus componentes funcionais, protocolos e interfaces, bem como suas interações.

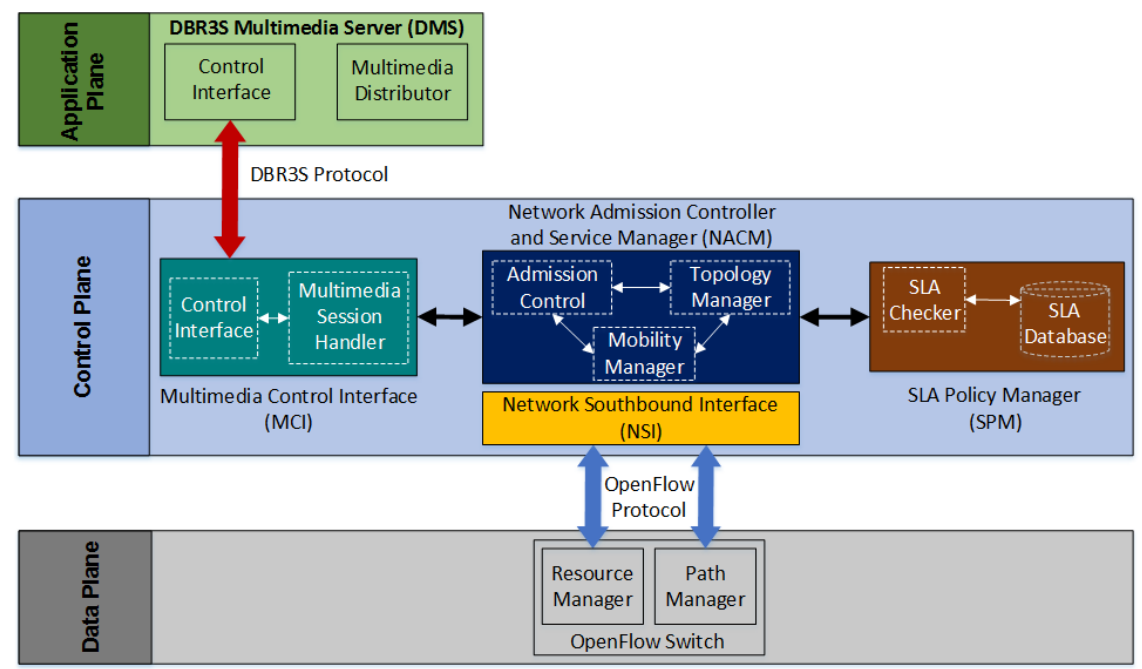

Figura 1. Arquitetura DBR3S

O controlador DBR3S é responsável por realizar as seguintes tarefas: $(i)$ controle de admissão; (ii) alocação/realocação de recursos; (iii) gerência de mobilidade; (iv) verificação de SLAs; e (v) manter o DMS atualizado acerca dos recursos disponíveis. De modo a prover suporte a tais funcionalidades, o DBR3S adota três componentes chave: $(i)$ Network Admission Controller and Service Manager (NACM), responsável por realizar a verificação da disponibilidade dos recursos de rede para uma nova requisição, aplicar as decisões baseadas em SLA nos elementos de interconexão, bem como executar o gerenciamento de mobilidade; (ii) SLA Policy Manager (SPM), responsável pelo armazenamento das definições de SLA e as devidas verificações durante uma nova admissão; e (iii) Multimedia Control Interface (MCI), que provê a interface primária entre o controlador DBR3S e o DMS para lidar com as requisições multimídia.

O DMS é responsável por realizar a entrega do conteúdo multimídia aos seus clientes, adaptando a qualidade da transmissão se necessário, sem interrupções. As adaptações são realizadas de acordo com as medições sobre os recursos disponíveis reali- 
zadas pelo plano de controle, que auxilia o DBR3S fornecendo a quantidade de largura de banda disponível para uma sessão multimídia por meio das sinalizações do DBR3S Protocol (DBR3SP). Esse protocolo também permite ao DMS negociar e gerenciar sessões multimídia com o controlador DBR3S. Mais detalhes sobre a arquitetura desse sistema, bem como seus procedimentos para a configuração de sessões multimídia e as mensagens de operação do DBR3SP podem ser encontradas em [Santos et al. 2017].

\section{Avaliação e resultados}

A avaliação do sistema é baseada no testbed ilustrado na Figura 2, composto de 6 PoAs e 9 switches virtuais (Open vSwitch ${ }^{1}$ ) com suporte a OpenFlow v1.3, 1 controlador SDN baseado no framework $\mathrm{Ryu}^{2}, 93$ MOs distribuídos nos 6 PoAs disponíveis, e um servidor multimídia (DMS) conectado a um nó de entrada.

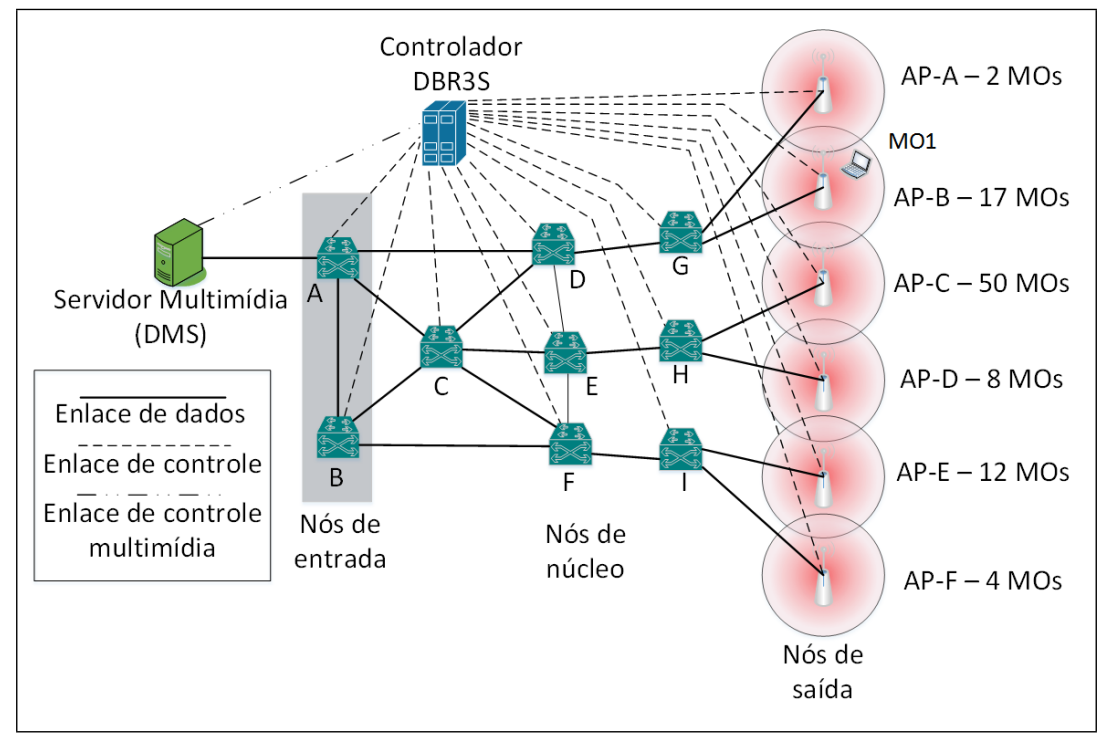

Figura 2. Topologia do testbed

A infraestrutura SDMN do testbed é virtualizada através ferramenta Mininet-Wifi [Fontes et al. 2015], que permite a emulação do meio wireless 802.11 e provê pacotes Traffic Control (TC), com suporte a escalonamento de filas Hierarquical Token Bucket (HTB), de modo a habilitar rotas com QoS baseado em classes. A distribuição do vídeo é baseada na ferramenta Evalvid [Klaue et al. 2003], modificada para suportar transmissão de vídeo adaptativa do DBR3S.

De modo a determinar a viabilidade do DBR3S, o testbed avalia a performance de uma sessão multimídia sob saturação de recursos. Para esse fim, cada um dos 93 MOs recebe uma sessão multimídia do DMS, sendo que cada MO procura receber o conteúdo na máxima qualidade disponível, estando sujeito às variações nas condições da rede e nas definições de SLA sob a qual um cliente está condicionado. Os MOs presentes na rede representam usuários que estão distribuídos em diferentes níveis de serviço (i.e. Ouro, Prata e Bronze).

\footnotetext{
${ }^{1}$ http://openvswitch.org/

${ }^{2}$ https://osrg.github.io/ryu/
} 


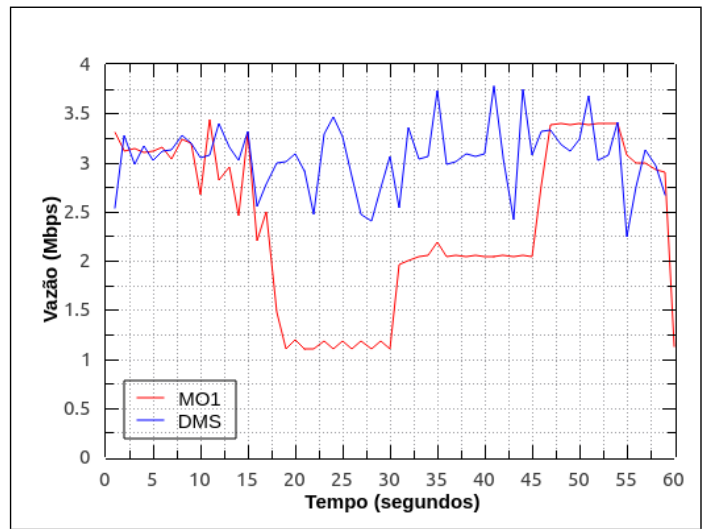

(a)

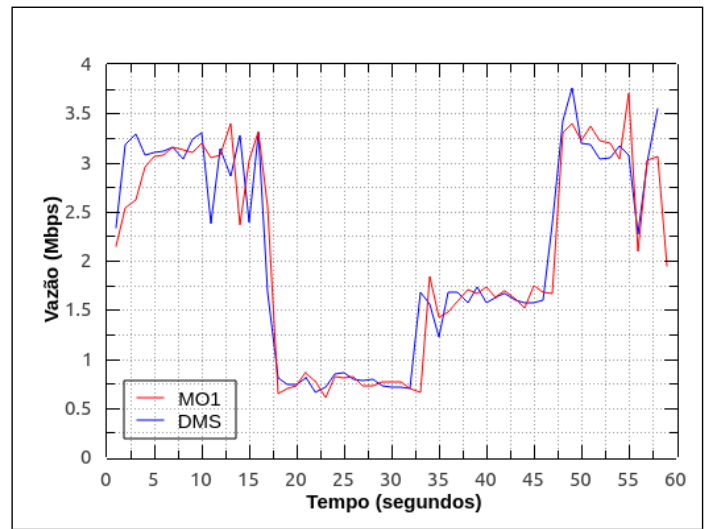

(b)

Figura 3. Comparativo entre a vazão do servidor DMS e do MO1 sem (a) e com (b) o mecanismo de controle de qualidade do DBR3S

Cada conjunto de avaliação possui a duração de 45 segundos, consistindo num MO (MO1) (conforme a Figura 2) que recebe o vídeo do DMS enquanto se move da área de cobertura do AP-B para o AP-D. Os resultados apresentados na Figura 3 revelam a taxa de transmissão (vazão) dos dados enviados pelo DMS e recebidos pelo MO1. O primeiro conjunto de avaliação é realizado com o modelo de sobre-provisionamento de recursos baseado em políticas, no entanto sem o mecanismo de adaptação da qualidade do vídeo. Devido a isso, como mostrado na Figura 3(a), a vazão enviada do DMS para o MO1 se mantém em aproximadamente $3 \mathrm{Mbps}$ durante todo o experimento.

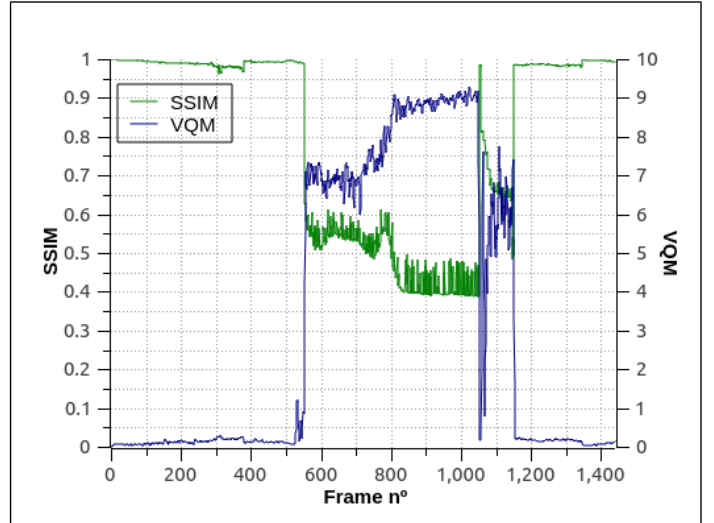

(a)

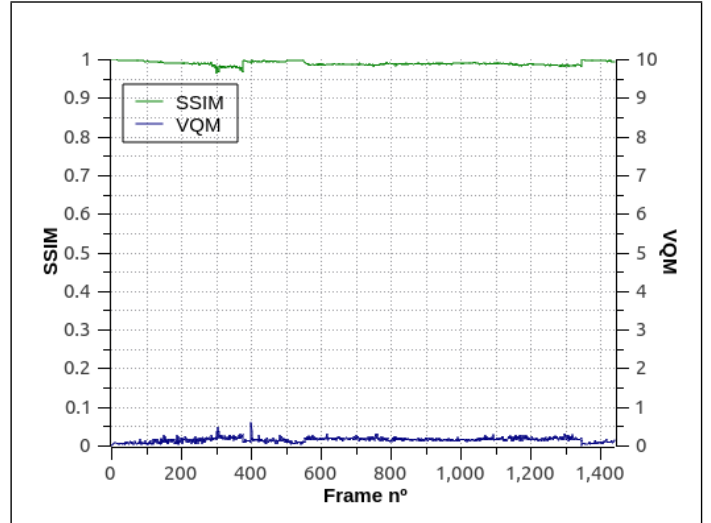

(b)

Figura 4. Resultado da avaliação do mecanismo de adaptação DBR3S com as métricas SSIM e VQM

No momento do primeiro handover (instante 15s), a largura de banda disponível para o MO1 é de aproximadamente $1 \mathrm{Mbps}$. Isso é provocado pelas 50 sessões multimídia não-adaptativas que são conectadas por meio de um enlace de $54 \mathrm{Mbps}$, levando a um severo congestionamento. Visto que o MO1 pertence ao nível de serviço Bronze, a quantidade de recursos disponíveis durante o período de $15 \mathrm{~s}$ a 30 s não viola o SLA, de modo que haja uma garantia de pelo menos $0.75 \mathrm{Mbps}$ de largura de banda. Entretanto, isso leva a uma queda significativa na qualidade do conteúdo transmitido, como mostrado na Figura 4(a), onde a QoE percebida pelo MO é excessivamente prejudicada devido a 
ausência de recursos, segundo confirmação dos níveis das métricas de Similaridade Estrutural (Structural Similarity - SSIM) e Métrica de Qualidade de Vídeo (Video Quality Metric-VQM).

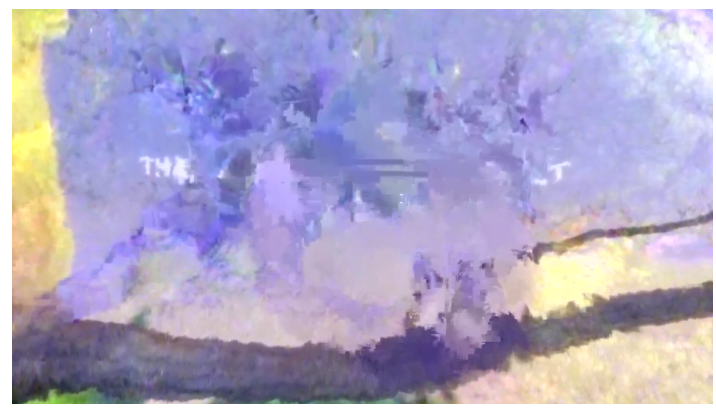

(a)

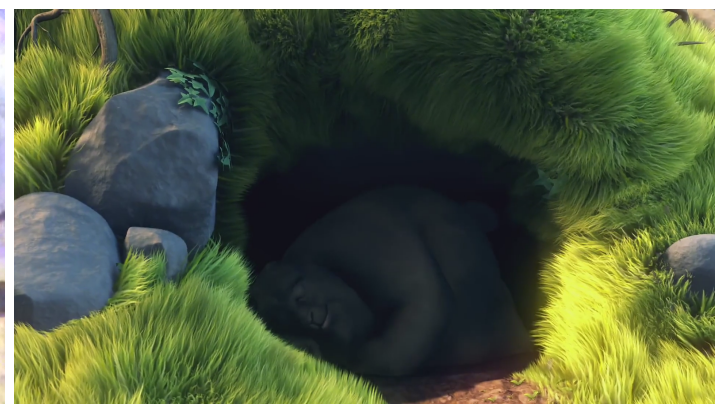

(b)

Figura 5. Comparação de quadros em sessões sem (a) e com (b) o DBR3S

No segundo conjunto de avaliação, com o mecanismo de controle de qualidade do DBR3S, os resultados da Figura 3(b) revela que os valores da vazão do tráfego enviado pelo servidor ao MO1 acompanha a taxa de quantidade de dados recebida por ele durante toda a avaliação. O controlador DBR3S foi capaz de ajustar, nos períodos de congestionamento, a taxa de transmissão das sessões multimídia para que o MO1 pudesse ser acomodado nos PoAs AP-C e AP-D. Tais ajustes permitiram a manutenção de bons níveis de QoE em uma transmissão sem interrupções e sem perdas significativas na qualidade do vídeo, como mostra a Figura 4(b).

A Figura 5 mostra a perspectiva do usuário sobre o conteúdo recebido. O quadro exibido nas duas avaliações é extraído em um momento randômico entre o primeiro e o segundo handover (quadro 923). Esse resultado, bem como os anteriores, demonstram que o DBR3S foi capaz de melhorar os níveis de QoE do usuário, adotando um mecanismo de controle de qualidade baseado nas estimativas realizadas pelo controlador SDMN ao invés da abordagem tradicional cujos mecanismos residem nos MOs. As ações tomadas pelo controlador DBR3S, em conjunto com o DMS, confirmam a viabilidade do sistema e da arquitetura proposta.

\section{Conclusão}

Neste trabalho apresentamos o Dynamic Bit Rate Streaming Service over SDMN (DBR3S), um sistema que estende as capacidades do plano de controle SDMN para permitir o fornecimento de um serviço multimídia com controle de qualidade otimizado. Para isso, o DBR3S transfere as funções de estimativas de recursos disponíveis para o controlador, que possui visão e controle holístico da rede. Além disso, as adaptações das sessões multimídia são aplicadas diretamente no servidor, que é atualizado em tempo real pelo plano de controle por meio do DBR3SP sobre as condições da rede.

Os trabalhos futuros do DBR3S consistem em estender as capacidades de decisão de adaptação multimídia por meio de uma abordagem baseada em Mobile Edge Computing (MEC), bem em empreender uma avaliação em cenários de larga escala composto por hardware real e com diferentes padrões de mobilidade e condições da rede. 


\section{Referências}

Akhshabi, S., Anantakrishnan, L., Begen, A. C., and Dovrolis, C. (2012). What happens when HTTP adaptive streaming players compete for bandwidth? In Proceedings of the 22nd international workshop on Network and Operating System Support for Digital Audio and Video, pages 9-14. ACM.

Chen, Y., Wu, K., and Zhang, Q. (2015). From QoS to QoE: A tutorial on video quality assessment. IEEE Communications Surveys \& Tutorials, 17(2):1126-1165.

Fontes, R., Afzal, S., Brito, S., Santos, M., and Rothenberg, C. (2015). Mininet-WiFi: Emulating software-defined wireless networks. In 2015 11th International Conference on Network and Service Management (CNSM), pages pp. 384-389.

Ganchev, I., Curado, M., and Kassler, A. (2014). Wireless Networking for Moving Objects: Protocols, Architectures, Tools, Services and Applications, volume 8611. Springer.

Gupta, P. K., Rajakumar, R., and Kumar, C. (2012). Analysis of impact of network activity on energy efficiency of 3GPP-LTE. In India Conference (INDICON), 2012 Annual IEEE, pages 665-669. IEEE.

Haleplidis, E., Pentikousis, K., Denazis, S., Salim, J. H., Meyer, D., and Koufopavlou, O. (2015). Software-Defined Networking (SDN): Layers and Architecture Terminology. Internet engineering task force (ietf) request for comments (rfc) número 7426.

Klaue, J., Rathke, B., and Wolisz, A. (2003). EvalVid - A Framework for Video Transmission and Quality Evaluation. In In Proc. of the 13th International Conference on Modelling Techniques and Tools for Computer Performance Evaluation, pages 255272.

Liyanage, M., Gurtov, A., and Ylianttila, M. (2015). SDMN, pages 440-. Wiley Telecom.

Rebay, V. (2011). Mobility and Multihoming Possibilities in Network Layer, Using Smartphones. In IEEE 12th International Symposium on Computational Intelligence and Informatics (CINTI), pages pp. 573-578.

Santos, C. H., Silva, F. S. D., and Neto, A. J. V. (2017). An innovative dynamic bit rate streaming approach to improve mobile user multimedia quality of experience. In Proceedings of the 15th ACM International Symposium on Mobility Management and Wireless Access, MobiWac '17, pages 1-8, New York, NY, USA. ACM.

Seufert, M., Egger, S., Slanina, M., Zinner, T., Hoßfeld, T., and Tran-Gia, P. (2015). A Survey on Quality of Experience of HTTP Adaptive Streaming. IEEE Communications Surveys Tutorials, 17(1):469-492.

Silva, F., Castillo-Lema, J., Neto, A., Silva, F., Rosa, P., Corujo, D., Guimarães, C., and Aguiar, R. (2014). Entity title architecture extensions towards advanced qualityoriented mobility control capabilities. In Computers and Communication (ISCC), 2014 IEEE Symposium on, pages 1-6. IEEE.

Strassner, J. (2003). Policy-based network management: solutions for the next generation. Morgan Kaufmann. 